Research Paper

\title{
Trend Analysis of Primary Midwife-led Delivery Care at a Japanese Perinatal Center
}

\author{
Shunji Suzuki ${ }^{\bowtie}$ \\ Department of Obstetrics and Gynecology, Japanese Red Cross Katsushika Maternity Hospital, Tokyo, Japan.
}

$\triangle$ Corresponding author: Shunji Suzuki, MD. Department of Obstetrics and Gynecology, Japanese Red Cross Katsushika Maternity Hospital, 5-11-12 Tateishi, Katsushika-ku, Tokyo 124-0012, Japan. E-mail: czg83542@mopera.ne.jp Tel: +81-3-3693-5211 Fax: +81-3-3694-8725.

(c) Ivyspring International Publisher. This is an open-access article distributed under the terms of the Creative Commons License (http://creativecommons.org/ licenses/by-nc-nd/3.0/). Reproduction is permitted for personal, noncommercial use, provided that the article is in whole, unmodified, and properly cited.

Received: 2013.II.23; Accepted: 2014.02.27; Published: 2014.03.15

\begin{abstract}
Objective: To perform trend analysis of primary midwife-led delivery care for 'low risk' pregnant women at our hospital.

Methods: A retrospective cohort study was performed to examine trends and outcomes of labor under primary midwife-led delivery care at the Japanese Red Cross Katsushika Maternity Hospital between 2008 and 2012.

Results: During the study period, the rate of deliveries initially considered 'low risk' decreased from 25 to $22 \%(p<0.01)$. This change was associated with increased cases of previous Cesarean deliveries and preterm delivery. There were no significant changes in the neonatal outcomes; however, the rate of Cesarean delivery and incidence of severe perineal laceration in primary midwife-led delivery care were decreased from 2.1 and $3.3 \%$ to $0.3(p=0.02)$ and $\mathrm{I} .1 \%(p=0.04)$, respectively due to the close cooperation between midwives and obstetricians.

Conclusion: The rate of deliveries initially considered 'low risk' decreased over the last 5-year period. Closer cooperation between midwives and obstetricians is important in primary midwife-led delivery care.
\end{abstract}

Key words: Midwife-led care, obstetric shared care, referral, Japan.

\section{Introduction}

Although midwifery care for 'low risk' pregnant women during labor has been reported to be associated with various advantages, such as increased rates of high maternal satisfaction and a decrease in unnecessary medical interventions, ${ }^{1-9}$ in Japan, most deliveries are managed by obstetricians in a hospital or private obstetric clinic, where the autonomy of midwives tends to be limited. In Japan, there is a system of Nurse Midwifery and, although midwives legally have the right to practice autonomously, in reality, this seems not to be the case. ${ }^{1,2}$ To maintain the safety of primary midwife-led delivery care, good communication and cooperation between obstetricians and midwives is needed. This is because, if complications occur or threaten to occur during the primary mid- wife-led delivery care, the midwives have to refer the woman to obstetricians at the same or a neighboring hospital or private obstetric clinic as soon as possible.7, 11,12 In cases managed by independent midwives in Japan, in addition, many intervention measures, such as oxytocin infusion, epidural anesthesia, episiotomy, and instrumental delivery, are not available based on Japanese legal restrictions. In our earlier observations, however, there was no evidence that primary midwife-led care is unsafe for 'low risk' pregnant women compared with obstetric shared care at our hospital, which is one of main perinatal centers in Tokyo, Japan (about 2,000 deliveries per year). ${ }^{10-13}$ In these studies, in addition, about $85 \%$ of 'low risk' pregnant women requested to give birth under midwifery care.10,11 
Therefore, safe midwifery care with the backup of obstetricians may be required for 'low risk' pregnant women in Japan. This study involved trend analysis of primary midwife-led delivery care for 'low risk' pregnant women at our hospital.

\section{Methods}

In this study, we examined the trends regarding 'low risk' pregnant women under primary midwife-led delivery care at the Japanese Red Cross Katsushika Maternity Hospital between 2008 and 2012. The protocol for this analysis was approved by the Ethics Committee of the Japanese Red Cross Katsushika Maternity Hospital. In addition, informed consent for analysis from a retrospective database was obtained from each subject during their hospital visit.

In our hospital, pregnant women who are initially considered 'low risk' can choose freely between midwife-led care and obstetric shared care when they are admitted at the spontaneous onset of labor pains and/or rupture of the membranes at 37-41 weeks' gestation, as reported previously. ${ }^{10-13}$ In the midwife-led care unit, midwives can practice autonomously and are fully accountable for their own practice, unsupervised by obstetricians. As a general rule of our midwife care unit, one pregnant woman is managed by three staff midwives, while, in our shared unit, one pregnant woman is managed by one or two staff obstetricians and two staff midwives over a 24-hour period. In cases managed by independent midwives, many intervention measures, such as oxytocin infusion, epidural anesthesia, episiotomy, and instrumental delivery, are not available based on Japanese legal restrictions. In our hospital, the fetal heart rate is monitored intermittently with a cardiotocogram during the first stage of labor without the augmentation of labor pains. The fetal heart rate is monitored continuously during the first stage of labor with the augmentation of labor pains, and during the second stage of labor. Furthermore, epidural anesthesia is not performed for 'low risk' pregnant women in our hospital.

Factors used to exclude women from the 'low risk' group include the following: ${ }^{10,11}$ (1) medical history: pregnancy induced hypertension, chronic hypertension, diabetes mellitus, renal disease, idiopathic thrombocytopenia, and other systemic illnesses; (2) gynecological history: history of infertility therapies of in vitro fertilization, congenital uterine anomalies, uterine myomatosus, and adnexal anomaly; (3) obstetric history: narrowing of the pelvic outlet, cephalopelvic disproportion, previous Cesarean section, previous anal sphincter injury, previous postpartum hemorrhage $\geq 1,000 \mathrm{~mL}$ with blood transfusion, previous manual removal of placenta, previous gesta- tional diabetes, and history of severe preeclampsia; (4) complications during the present pregnancy: multiple pregnancy, nonvertex presentation, obesity (maternal body mass index before pregnancy $\geq 25$ and/or during the third trimester $\geq 28$ ), anemia (hemoglobin < $9.0 \mathrm{~g} / \mathrm{dl}$ ) , epilepsy with treatment, polyhydramnios, oligohydramnios, low set placenta, placenta previa, fetal growth restriction, heavy for dates fetus, gestational diabetes, and pregnancy induced hypertension. When risk factors are present, those women are managed by obstetricians and midwives; (5) complications during labor: intrauterine infection, thick meconium staining, prolongation of labor such as active-phase dilation $<1 \mathrm{~cm} /$ hour and duration of second stage of labor $\geq 2$ hours, prolonged rupture of membranes ( $\geq 24$ hours), uterine inertia, arrest of labor, and fetal heart rate abnormality such as non-reassuring fetal status. When these factors are present, the women are transferred to be managed mainly by obstetricians (obstetric shared care) in a standard Western-style delivery room or surgery room. During the study period, these criteria for the risk of delivery remained unchanged.

A retrospective cohort study was performed to examine trends and outcomes of labor under primary midwife-led delivery care. Factors related to patients and perinatal outcomes were as follows: maternal age, parity, gestational age at delivery, history of previous Cesarean delivery, rate of referral from midwifery to shared care, indications for referral, augmentation of labor pains, delivery mode, episiotomy, severe perineal laceration (perineal laceration either third- or fourth-degree laceration), postpartum hemorrhage, Apgar score, and umbilical artery $\mathrm{pH}$.

Statistical analyses were carried out using the statistical software SAS version 8.02 (SAS Institute, Cary, NC, USA), and differences with $p<0.05$ were considered significant. Linear regression was performed to estimate the trend over time for each factor, initially 'low risk' delivery rate for each factor, and obstetric outcomes.

\section{Results}

During the 5-year period, there were 9,687 women in singleton labor managed at $\geq 22$ weeks' gestation at the Japanese Red Cross Katsushika Maternity Hospital. Of these, 2,214 cases (23\%) were initially considered 'low risk' at admission based on our definition of 'low risk' explained in Methods.

Table 1 shows the changes in maternal characteristics of the singleton pregnancies, number of deliveries initially considered 'low risk', and maternal requests. During the study period, the rate of women with a history of previous Cesarean deliveries and preterm delivery increased from 11 and $8.8 \%$ to $14(p$ 
$=0.049)$ and $11 \%(p=0.047)$, respectively, while the rate of deliveries initially considered 'low risk' decreased from 25 to $22 \%(p<0.01)$. The decreased rate of deliveries initially considered 'low risk' seemed to be related to the increased rate of women having a history of previous Cesarean deliveries and preterm delivery. The rate of maternal requests to give birth under midwife care did not change significantly during the study period.

Table 2 shows the changes in maternal characteristics and obstetric outcomes under the primary midwife-led delivery care during the study period. There were no significant changes in the rate of the maternal characteristics and neonatal outcomes dur- ing the study period; however, the rate of Cesarean delivery and incidence of severe perineal laceration decreased from 2.1 and $3.3 \%$ to $0.3(p=0.02)$ and $1.1 \%$ $(p=0.04)$, respectively.

Table 3 shows the trends in referrals from primary midwife-led to shared care by parity during the study period. There were no significant changes in the rates of referral involving both nulliparous and parous women.

Table 4 shows the changes in the rates of the main 3 indications for referral from primary midwife-led to shared care. There were no significant changes in the rates of these indications during the study period.

Table I. Changes in maternal characteristics in singleton pregnancies, number of deliveries initially considered 'low risk', and maternal requests.

\begin{tabular}{|c|c|c|c|c|c|}
\hline Year & 2008 & 2009 & 2010 & 2011 & 2012 \\
\hline Total singleton deliveries & 1,960 & 2,050 & 1,889 & 1,844 & 1,944 \\
\hline Maternal age $\geq 35$ years & $683(35)$ & 725 (35) & $694(37)$ & $676(37)$ & $732(38)$ \\
\hline Nulliparity & $959(49)$ & $1,023(50)$ & $927(49)$ & $904(49)$ & $973(50)$ \\
\hline History of previous Cesarean delivery & $225(11)$ & $239(12)$ & $257(14)^{*}$ & $237(13)$ & $264(14)^{*}$ \\
\hline Preterm deliveries & $173(8.8)$ & $170(8.3)$ & $199(11)$ & $176(9.5)$ & $208(11)^{*}$ \\
\hline Initially 'low risk' deliveries & $497(25)$ & $455(22)$ & $438(23)$ & $394(21)^{*}$ & $430(22)^{*}$ \\
\hline \multicolumn{6}{|l|}{ Maternal requests } \\
\hline Shared care & $77(15)$ & $69(15)$ & $51(16)$ & $44(12)$ & $74(17)$ \\
\hline Midwifery care & $420(85)$ & $386(85)$ & $368(84)$ & $350(88)$ & $356(83)$ \\
\hline
\end{tabular}

Values are presented as number (percentage).

${ }^{*} P<0.05$ vs. 2008 .

Table 2. Changes in maternal characteristics and obstetric outcomes under primary midwife-led delivery care during the study period.

\begin{tabular}{|c|c|c|c|c|c|}
\hline Year & 2008 & 2009 & 2010 & 2011 & 2012 \\
\hline Total & 420 & 386 & 368 & 350 & 356 \\
\hline Maternal age $\geq 35$ years & $135(32)$ & $122(32)$ & $110(30)$ & $108(31)$ & $110(31)$ \\
\hline Nulliparity & $202(48)$ & $187(48)$ & $159(43)$ & $167(47)$ & $164(46)$ \\
\hline Referrals & $189(43)$ & $159(41)$ & $179(49)$ & $160(46)$ & $167(47)$ \\
\hline Oxytocin use & $76(18)$ & $56(15)$ & $82(22)$ & $55(16)$ & $61(17)$ \\
\hline Episiotomy & $99(24)$ & 73 (19) & $64(18)$ & $88(25)$ & $75(21)$ \\
\hline Vacuum/forceps deliveries & $28(6.7)$ & $20(5.2)$ & $15(4.1)$ & $18(5.1)$ & $17(4.8)$ \\
\hline Cesarean deliveries & $9(2.1)$ & $8(2.1)$ & $10(2.7)$ & $4(1.1)$ & $1(0.3)$ \\
\hline Umbilical artery $\mathrm{pH}<7$ & $2(0.5)$ & $3(0.8)$ & $3(0.8)$ & $0(0)$ & $0(0)$ \\
\hline Apgar score at $5 \mathrm{~min}<4$ & $0(0)$ & $0(0)$ & $1(0.3)$ & $1(0.3)$ & $1(0.3)$ \\
\hline Severe perineal laceration & $14(3.3)$ & $6(1.5)$ & $7(2.0)$ & $1(0.3)$ & $4(1.1)$ \\
\hline Postpartum hemorrhage $\geq 1,000 \mathrm{ml}$ & $21(5.0)$ & $14(3.6)$ & $21(5.7)$ & $18(5.1)$ & $18(5.0)$ \\
\hline
\end{tabular}

Values are presented as number (percentage).

${ }^{*} P<0.05$ vs. 2008.

Table 3. Trends in referrals from primary midwife-led to shared care by parity during the study period.

\begin{tabular}{|c|c|c|c|c|c|}
\hline Year & 2008 & 2009 & 2010 & 2011 & 2012 \\
\hline \multicolumn{6}{|l|}{ Nulliparous women } \\
\hline Total primary midwife-led care & 202 & 187 & 159 & 167 & 164 \\
\hline Referrals & 125 & 108 & 115 & 109 & 111 \\
\hline Rate of referral (\%) & 62 & 58 & 72 & 65 & 68 \\
\hline \multicolumn{6}{|l|}{ Multiparous women } \\
\hline Total primary midwife-led care & 218 & 199 & 209 & 51 & 192 \\
\hline Referrals & 55 & 41 & 64 & 13 & 56 \\
\hline Rate of referral (\%) & 25 & 21 & 31 & 28 & 29 \\
\hline
\end{tabular}

${ }^{*} P<0.05$ vs. 2008. 
Table 4. Changes in rates of the main three indications for referrals from primary midwife-led to shared care.

\begin{tabular}{|c|c|c|c|c|c|}
\hline Year & 2008 & 2009 & 2010 & 2011 & 2012 \\
\hline Total & 189 & 159 & 179 & 160 & 167 \\
\hline Fetal heart rate abnormality & $57(32)$ & $57(36)$ & $50(28)$ & $56(35)$ & $58(35)$ \\
\hline Failure to progress & $44(24)$ & $41(26)$ & $52(29)$ & $41(26)$ & $46(28)$ \\
\hline Prolonged rupture of membranes ( $\geq 24$ hours) & $13(7.2)$ & $12(7.5)$ & $11(6.1)$ & $10(6.3)$ & $10(6.0)$ \\
\hline
\end{tabular}

Values are presented as number (percentage).

$* P<0.05$ vs. 2008.

\section{Discussion}

Our obstetric care system involves the division of women in labor into low and high risk groups. The women who are initially considered low risk can choose freely between midwife-led care and obstetric shared care. If complications occur or risk factors arise during labor in the primary midwife-led care, they are referred to obstetric shared care.

Based on the current observations, the rate of low risk delivery in our hospital has decreased significantly over the last 5 -year period, even though the criteria for the risk of delivery remained unchanged. Considering the increased rate of high maternal satisfaction under midwife-led care, ${ }^{1-9}$ the current results are very disappointing. These observations seem to be associated with the increased rate of high risk deliveries, such as cases involving previous Cesarean delivery and preterm labor. Most of these high risk pregnant women were referred to our hospital from private obstetric clinics during pregnancy. In Japan, half of all pregnant women and their deliveries are now managed at private obstetric clinics which only manage 'low risk' pregnant women, while most 'high risk' pregnant women are managed at large scale perinatal centers or general hospitals. This situation may introduce a serious bias in obstetric studies carried out in Japan; it may appear that, at a global level, the birth process is becoming more medicalized in Japan.

In this study, about $85 \%$ of 'low risk' pregnant women requested to give birth under midwifery care over the study period. These findings support our previous studies ${ }^{10,11}$, and they indicate that Japanese pregnant women are more likely to be highly satisfied with delivery under midwife-led delivery care.

In this study, the rate of Cesarean delivery and incidence of severe perineal laceration were significantly decreased during the 5-year period. These changes indicate that maternal comfort has been increased in various modes of delivery. The reasons for this are not clear because the criteria for transfer from midwife-led to shared care have not been changed; however, some possible reasons can be proposed. One reason may be the small sample size of this study. During the study period, for example, Cesarean de- liveries in this study have been numbered 1-9 cases per year. Thus, statistical results in this study would change with an increase or decrease of 1-2 cases. Another reason may be that the cooperation between midwives and obstetricians in our hospital became closer. Delayed collaboration and/or transfer between midwifery and obstetric care have been reported to cause adverse perinatal outcomes. ${ }^{14,15}$ Although the current protocol in our hospital had been deemed safe, by referring to these previous reports, we reviewed cases in our primary midwifery care every month during the study period. We reviewed our management of 'low risk' pregnant women during labor in order to decrease the incidence of unnecessary abnormal deliveries. Although the criteria for the risk of delivery remained unchanged, the timing of our discussion about each delivery of 'low risk' pregnant woman likely to transfer has become earlier. The obstetricians became to be able to have the information of the deliveries before their transfers. In addition, we began to endeavor mental care of the 'low risk' pregnant women to accept their transfers easier. Such examinations might lead to a reduced incidence of severe perineal laceration or Cesarean section.

Although there has been no evidence that the primary midwife-led care is unsafe for 'low risk' pregnant women compared with obstetric shared care, and midwife-led care has been reported to be associated with increased maternal satisfaction, the rate of 'low risk' pregnancy has decreased significantly over the last 5-year period. Midwife-led care may be recommended for 'low risk' pregnant women; however, its role and effect should be re-examined frequently. Close cooperation between midwives and obstetricians is important in primary midwife-led delivery care.

\section{Competing Interests}

The authors have declared that no competing interest exists.

\section{References}

1. Misago C, Umenai T, Noguchi M, Mori T, Mori T. Satisfying birthing experiences in Japan. Lancet 2000; 355: 2256.

2. Page L. Human resources for maternity care: the present system in Brazil, Japan, North America, Western Europe and New Zealand. Int J Gynecol Obstet 2001; 75: S81-8. 
3. Hatem M, Sandall J, Devane D, Soltani H, Gates S. Midwife-led versus other models of care for childbearing women. Cochrane Database Syst Rev. 2008;: CD004667.

4. Hundley VA, Cruickshank FM, Lang GD, Glazender CMA, Milne JM, Turner M, Blyth D, Mollison J, Donaldson C. Midwife managed delivery unit: a randmised controlled comparison with consultant led care. BMJ 1994; 309: 1400-4.

5. Turnbull D, Holmes A, Shields N, Cheyne H, Twaddle S, Gilmour WH, McGinley M, Reit M, Johnstone I, Geer I, Mcllwain G, Lunan CB. Randmised, controlled trial of efficiency of midwife-managed care. Lancet 1996; 348: 213-8.

6. MacVicar J, Dobbie G, Owen-Johnstone L, Jagger C, Hopkins M, Kennedy J. Simulated home delivery in hospital: a randomized controlled trial. Br J Obstet Gynaecol 1993; 100: 316-23.

7. Amelink-Verburg MP, Rijnders MEB, Buitendijik SE. A trend analysis in referrals during pregnancy and labor in Dutch midwifery care 1988-2004. BJOG 2009; 116: 923-32.

8. Campbell R, Macfarlance A, Hempsall V, Hatchard K. Evaluation of midwife-led care provided at the Royal Bournemouth Hospital. Midwifery 1999; 15: 183-93.

9. Christiaens W, Gouwy A, Bracke P. Does a referral from home to hospital affect satisfaction with childbirth? A cross-national comparison. BMC Health Service Research 2007; 7: 109.

10. Suzuki S, Satomi M, Miyake H. Referrals during labor in midwifery care. J Nippon Med Sch 2009; 76: 226-8.

11. Suzuki S, Hiraizumi Y, Satomi M, Miyake H. Midwife-led care unit for 'low risk' pregnant women in a Japanese hospital. J Matern Fetal Neonatal Med 2011; 24: 1046-50.

12. Suzuki S. Obstetric outcomes of low-risk labors at 'Japanese tatami' mat delivery room: a preliminary study. J Perinat Med 2009; 37: 709-11

13. Suzuki S. The attitude of 'low risk' pregnant women in Japan toward midwife-led care during pregnancy and labor. J Matern Fetal Neonatal Med. 2012; 25: 2141-2.

14. Evers AC, Brouwers HA, Hukkelhoven CW, Nikkels PG, Boon $\mathrm{J}$, van Egmond-Linden A, Hillegersberg J, Snuif YS, Sterken-Hooisma S, Bruinse HW, Kwee A: Perinatal mortality and severe morbidity in low and high risk term pregnancies in theNetherlands: prospective cohort study. BMJ. 2010; 341: c5639.

15. Vandenbroucke JP. Dutch perinatal mortality. Study did a good job. BMJ. 2010; 341: c7042. 